DNA REPAIR

\section{The guardian}

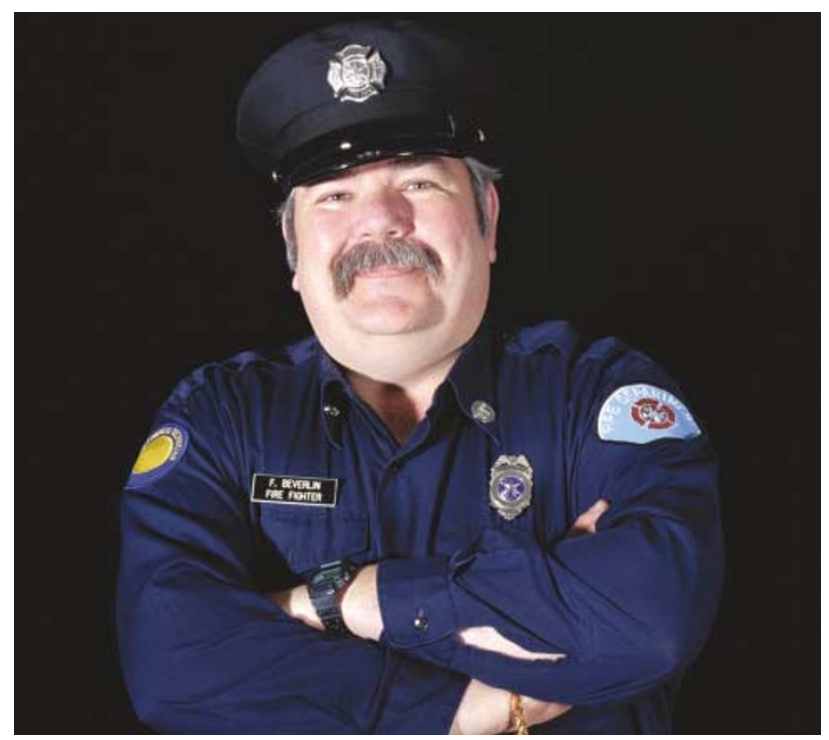

Artemis is the Greek goddess that guards young children and small animals. The gene Artemis is therefore appropriately named, as it seems to be a genome guardian that protects children and mice from cancer. In the February issue of the Journal of Clinical Investigation, Moshous et al. associate mutations in Artemis with immunodeficiency and predisposition to lymphoma.

Artemis was initially identified as a factor involved in the non-homologous end-joining (NHEJ) phase of $\mathrm{V}(\mathrm{D}) \mathrm{J}$ recombination of $\mathrm{T}$ - and B-cell receptor genes. Disruption of this gene in mice resulted in a complete absence of $\mathrm{T}$ and $\mathrm{B}$ lymphocytes, as well as radiosensitivity.

Moshous et al. studied the effects of null and hypomorphic Artemis mutations in four patients from two families. These patients were born with radiosensitive severe combined immunodeficiency (RS-SCID), which is characterized by an absence of both mature B and T lymphocytes and gamma-ray sensitivity. After the appearance of lymphocytopaenia during infancy, the children developed severe infections, and two of the four developed B-cell lymphomas. Their peripheral blood lymphocytes were found to have chromosome instabilities - particularly in chromosomes where Ig and T-cell receptor genes were located. So, do these lymphocytes have defects in NHEJ DNA-repair machinery?

Moshous et al. found that $\mathrm{T}$ cells from these patients had a virtual absence of $\mathrm{N}$ nucleotide additions at the $\mathrm{V}(\mathrm{D}) \mathrm{J}$ junctions. $\mathrm{N}$ regions are non-templated nucleotides that are added to the $3^{\prime}$ ends of RAG-mediated double-strand breaks by the enzyme terminal deoxynucleotidyl transferase (TdT). $\mathrm{N}$-region addition is known to require the NHEJ protein KU80, and these findings indicate that Artemis is also involved. Moshous et al. were able to complement the recombination

\title{
Making sense of missense
}

BRCA1 mutations have been linked to an increased risk of breast and ovarian cancer. Most of the highly penetrant alleles that have been identified encode truncated proteins, but missense alleles have also been recorded - their impact on cancer susceptibility is particularly difficult to assess because of low penetrance and the lack of adequate functional assays. In their paper, Fleming et al. describe an evolutionary approach to identify the missense alleles that are most likely to be associated with a disease phenotype.

The rationale behind this study is that mutations in functionally important amino acids are most likely to be associated with an increased risk of cancer. These key amino acids can be identified on the basis of their conservation in mammals or from evidence of recent positive selection in the human lineage. To find conserved BRCA1 regions, the authors aligned GenBank sequences for exon 11 of $B R C A 1$ from 57 mammals. Considering a region of five amino acids in length at a time, regions were defined as conserved if at least $80 \%$ of the sites were fixed or conserved (that is, identical in all species or all residues sharing similar biochemical properties, respectively), including the first and last residues. Seven out of eight conserved regions were located in regions known to interact with other proteins. In addition, a conserved stretch of amino acids was identified in a region of unknown function that is also conserved in $B R C A 1$ homologues from Xenopus and chicken.

If site conservation is a good indicator of its functional importance, it would be predicted that missense mutations affecting fixed sites, or resulting in non-conservative

substitutions at conservative sites, are most likely to be associated with a disease phenotype. In fact, 38 of the 139 documented missense alleles in exon 11 fall into one of these categories. The authors argue that these sites should be the priority for future studies, along with three missense mutations in residues that show signs of recent positive selection in human and primate lineages. Interestingly, these three amino acids lie in the region of BRCA1 that interacts with
RAD51 - a protein that is involved in DNA double-strand-break repair.

The authors present a promising approach for prioritizing the study of missense mutations in BRCA1, as well as in other genes that are associated with heritable diseases. Indeed, the authors show that this method can be used to predict the $\beta$-globin amino-acid residues that, when mutated, are associated with various globin pathologies. The true test of this approach, however, will be to determine how many of the 41 missense mutations that have been highlighted by Fleming et al. are associated with an increased risk of breast and ovarian cancer. Catherine Baxter, Associate Editor Nature Reviews Genetics

(0) References and links ORIGINAL RESEARCH PAPER Fleming, M. A. et al. Understanding missense mutations in the BRCA1 gene: an evolutionary approach. Proc. Natl Acad. Sci. USA 16 Jan 2003 (doi:10.1073/pnas.0237285100)

FURTHER READING Narod, S. A. Modifiers of risk of breast and ovarian cancer. Nature Rev. Cancer 2, 113-123 (2002) WEB SITE

Elaine A. Ostrander's lab: http://www.gs.washington. edu/faculty/ostrander.htm 